\title{
Evolutionary engineering improves tolerance for medium-chain alcohols in Saccharomyces cerevisiae
}

\author{
Stephanie A. Davis López ${ }^{1}$, Douglas Andrew Griffith² ${ }^{2}$ Brian Choi ${ }^{3}$, Jamie H. D. Cate ${ }^{4}$ \\ and Danielle Tullman-Ercek ${ }^{2^{*}}$ (1)
}

\begin{abstract}
Background: Yeast-based chemical production is an environmentally friendly alternative to petroleum-based production or processes that involve harsh chemicals. However, many potential alcohol biofuels, such as $n$-butanol, isobutanol and $n$-hexanol, are toxic to production organisms, lowering the efficiency and cost-effectiveness of these processes. We set out to improve the tolerance of Saccharomyces cerevisiae toward these alcohols.
\end{abstract}

Results: We evolved the laboratory strain of S. cerevisiae BY4741 to be more tolerant toward $n$-hexanol and show that the mutations which confer tolerance occur in proteins of the translation initiation complex. We found that $n$-hexanol inhibits initiation of translation and evolved mutations in the a subunit of elF 2 and the $y$ subunit of its guanine exchange factor elF2B rescue this inhibition. We further demonstrate that translation initiation is affected by other alcohols such as $n$-pentanol and $n$-heptanol, and that mutations in the elF2 and elF2B complexes greatly improve tolerance to these medium-chain alcohols.

Conclusions: We successfully generated S. cerevisiae strains that have improved tolerance toward medium-chain alcohols and have demonstrated that the causative mutations overcome inhibition of translation initiation by these alcohols.

Keywords: Saccharomyces cerevisiae, Medium-chain alcohols, Biofuels, Translation initiation, elF2, elF2B, Alcohol tolerance

\section{Background}

The brewer's yeast Saccharomyces cerevisiae is the production organism of choice for the most prominent commercial biofuel, bioethanol. In recent years, there has been growing interest in using this yeast for the production of advanced higher chain alcohol biofuels, such as $n$-butanol [1-6] and isobutanol [7, 8], and for bio-alcohols with other uses such as the prenyl alcohols, farnesol, nerolidol, and geranylgeraniol, which are fragrance components of essential oils [9]. A major challenge when using S. cerevisiae to produce

\footnotetext{
*Correspondence: ercek@northwestern.edu

2 Department of Chemical and Biological Engineering, Northwestern University, 2145 Sheridan Road, Tech E-136, Evanston, IL 60208-3109, USA Full list of author information is available at the end of the article
}

bio-alcohols, however, is that many of these compounds are toxic to cells. Ethanol and other alcohols accumulate in the yeast plasma membrane increasing its fluidity, and altering its stability and structure [10, 11]. They severely perturb membrane function by increasing the proton permeability of the plasma membrane, which diminishes the proton-motive force available to drive secondary active solute and nutrient transporters [10-12]. In addition to this indirect effect on membrane protein function, ethanol inhibits water transport across the plasma membrane by a direct interaction with the aquaporin $A Q Y 1$ [10], and higher chained $n$-alcohols (from propanol to hexanol) are inhibitors of the multidrug-resistance pumps Pdr5p and Snq2p [13]. The increased membrane fluidity caused by alcohols also results in a loss of intracellular molecules such as 
ATP, RNA, proteins and phospholipids [12, 13]. Taken together, the various effects of alcohols on yeast cells lead to the impairment of essential physiological processes including cellular energy maintenance, growth inhibition, and even cell death. Understanding these effects is the next step toward engineering solutions to improve tolerance.

Fusel alcohols, such as $n$-butanol and isoamyl alcohol, are by-products of amino acid metabolism in S. cerevisiae, and a promising series of studies revealed that these alcohols inhibit translation initiation by targeting the eIF2B guanine nucleotide exchange factor (GEF) that recycles the eIF2 complex from a GDP- to a GTP-bound form that is competent for translation initiation $[14,15]$. eIF2 plays an important role in translation initiation as it brings the methionine-charged initiator tRNA to the $40 \mathrm{~S}$ subunit of the ribosome $[16,17]$. To explain the inhibition by fusel alcohols, Taylor et al. [14] suggested that the alcohols either bind directly to eIF2B, or alter as-yet unidentified posttranslational modifications, such as phosphorylation, which are critical to the translation initiation process [15]. Moreover, mutations in eIF2B can modulate this inhibition $[14,15]$.

Adaptive laboratory evolution (ALE) is increasingly used as a technique for untargeted strain optimization $[18,19]$ and was successfully employed to identify biological solutions to biofuel and alcohol toxicity in S. cerevisiae [11, 20-22]. Here we used ALE to identify genetic targets that can be exploited to alleviate medium-chain alcohol toxicity in S. cerevisiae. We subjected wild-type yeast to an ALE experiment by gradually increasing the $n$-hexanol concentration in the growth medium to enrich for $n$-hexanol-tolerant $S$. cerevisiae strains. After two 30-day rounds of evolution, we identified strains with significantly more $n$-hexanol tolerance. These evolved strains also showed greater tolerance to a wide range other medium-chain alcohols (from propanol to octanol). Genome sequencing of our tolerant strains revealed three interesting mutations in proteins crucial for translation initiation. We found a D77Y mutation in the eIF2 $\alpha$ subunit, Sui2p, a D85E mutation in the eIF2By subunit, Gcd1p, and a R56C mutation in the eIF2B $\beta$ subunit, Gcd7p. We further show that $n$-hexanol, like $n$-butanol and isoamyl alcohol [14, 15], inhibits translation initiation, and that individually reverse-engineering the D77Y mutation in eIF2 $\alpha$ and the D85E mutation in eIF2B $\gamma$ confers tolerance to $n$-hexanol by relieving this inhibition. The D85E mutation in eIF2By is the first mutation identified in this subunit that reduces sensitivity to alcohols, and the D77Y mutation in eIF2 $\alpha$ is the first mutation in eIF2 shown to mitigate alcohol-dependent translational inhibition. This is the first report of tolerance engineering to $n$-hexanol in S. cerevisiae.

\section{Methods}

Yeast strains, media, and molecular biology for generation of yeast strains

Saccharomyces cerevisiae strains and plasmids used in this study are listed in Table 1. All adaptive evolution experiments were performed in the background of

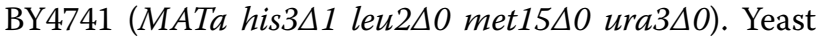
strains were grown in YPD medium [1\% yeast extract (Amresco), 2\% bacto-peptone (BD Chemical), 2\% dextrose (Amresco)] unless otherwise indicated.

Plasmid construction steps were performed using standard methods for recombinant DNA work [23] in E. coli strain DH10B and by in vivo homologous recombination (HR) in BY4741 [24]. BY4741 was transformed to $U R A^{+}, L E U^{+}$and $H I S^{+}$by a whole cell high-efficiency lithium acetate method [25]. To reverse engineer the mutant alleles $g c d 1-1, \operatorname{gcd} 7-2$, and sui2-2, these genes were cloned individually by HR onto low-copy CEN6/ ARS4 plasmids, and then individually or in combination transformed into BY4741 (see Table 1). Constructs and strains for the wild-type alleles were generated to serve as controls. The wild-type genes in the genome of transformed strains matching those on the plasmids were then deleted. To construct the plasmids, genomic DNA (gDNA) was isolated from strains sSD019 and sSD0021 with the YeaStar ${ }^{\mathrm{TM}}$ genomic DNA kit (Zymo Research, Irvine, CA), and used as a template to amplify gcd1-1, gcd7-2, and sui2-2 by PCR. Wild-type alleles for these genes were amplified from gDNA extracted from BY4741. All primer sequences used in this study can be found in Additional file 1: Table S1. The PCR primers added $38 \mathrm{bp}$ of sequence to the ends of the genes that are homologous to those flanking the SpeI and BamHI sites at the desired point of insertion in the destination vectors. Alleles $g c d 7-2$ and $G C D 7^{+}$were cloned into SpeI/BamHI-digested pAH005, which carries the LEU2 marker [26], to generate pSD469 and pSD474, respectively, and $g c d 1-1$, sui2-2, GCD $1^{+}$, and $S U I 2^{+}$were cloned into SpeI/BamHI-digested pAH056, which carries the URA3 marker [26] to create pSD450, pSD452, pSD442 and pSD444, respectively. Transformants were selected on solid synthetic dropout (SD) media (containing $20 \mathrm{~g} / \mathrm{l}$ agar) lacking uracil or leucine, or both $(0.67 \%$ yeast nitrogen base (BD Chemical), 2\% dextrose, $0.2 \%$ amino acid mix without uracil or leucine or both (US Biological). Recombinant plasmids were isolated using the QIAprep Spin Miniprep kit (Qiagen) [27], shuttled into E. coli, and the integrity of cloned sequences was confirmed by dyeterminator sequencing.

Gene deletions in strains carrying plasmids for wildtype and mutant alleles were performed using a cloningfree PCR-based allele replacement method [28]. The HIS3 selection marker from plasmid pML840 [26] was 
Table 1 List of Saccharomyces cerevisiae strains and plasmids used in this study and their genotypes

\begin{tabular}{|c|c|c|c|c|}
\hline Strain & Genotype & Plasmid & Plasmid description & Source \\
\hline BY4741 & MATa his $3 \Delta 1$ leu2 $\triangle 0$ met $15 \Delta 0$ ura $3 \Delta 0$ & None & & John Dueber ${ }^{\mathrm{a}}$ \\
\hline sSD003 & MATa his $3 \Delta 1$ leu2 $\triangle 0$ met15 $\triangle 0$ ura3 $\Delta 0$ gcd1-1 cit2-1 & None & & This study \\
\hline sSD006 & MATa his $3 \Delta 1$ leu $2 \Delta 0$ met $15 \Delta 0$ ura $3 \Delta 0$ gcd $7-2$ & None & & This study \\
\hline sSD019 & $\begin{array}{l}\text { MATa his } 3 \triangle 1 \text { leu } 2 \triangle 0 \text { met15 } \triangle 0 \text { ura3 } \triangle 0 \text { gcd1-1 cit2-1 pdr5-1 ubp13-1 } \\
\text { Isb6-1 nst 1-1 cox1-1 }\end{array}$ & None & & This study \\
\hline sSD021 & MATa his $3 \triangle 1$ leu2 $\triangle 0$ met15 $\triangle 0$ ura $3 \Delta 0$ gcd7-2 sui2-2 pdr5-2 sey1-2 & None & & This study \\
\hline sSD029 & MATa his $3 \Delta 1$ leu2 $\triangle 0$ met15 $\triangle 0$ ura3 $\triangle 0$ gcd1 $\triangle:: K a n M X 4$ & pSD450 & pAH056 CEN6/ARS4 URA3 gcd1-1 & This study \\
\hline sSD040 & MATa his $3 \Delta 1$ leu2 $\triangle 0$ met $15 \Delta 0$ ura $3 \Delta 0$ gcd $7 \triangle:$. His3 & pSD469 & pAH005 CEN6/ARS4 LEU2 gcd7-2 & This study \\
\hline sSD053 & MATa his $3 \Delta 1$ leu2 $\triangle 0$ met $15 \Delta 0$ ura $3 \Delta 0$ gcd $1 \Delta:: K a n M X 4$ & pSD442 & pAH056 CEN6/ARS4 URA3 GCD1 & This study \\
\hline sSD054 & MATa his $3 \Delta 1$ leu2 $\triangle 0$ met $15 \Delta 0$ ura $3 \Delta 0$ ged $7 \Delta:$. His3 & pSD474 & pAH005 CEN6/ARS4 LEU2 GCD7 & This study \\
\hline sSD055 & MATa his $3 \Delta 1$ leu2 $\triangle 0$ met $15 \Delta 0$ ura3 $\triangle 0$ sui2 $\triangle:: K a n M X 4$ & pSD444 & PAH056 CEN6/ARS4 URA3 SUI2 & This study \\
\hline sSD056 & MATa his $3 \Delta 1$ leu2 $\triangle 0$ met $15 \Delta 0$ ura3 $\triangle 0$ sui2 $\triangle:: K a n M X 4$ & pSD452 & pAH056 CENG/ARS4 URA3 sui2-2 & This study \\
\hline sSD058 & MATa his $3 \Delta 1$ leu2 $\triangle 0$ met15 $\triangle 0$ ura3 $\triangle 0$ sui2 $\triangle:: K a n M X 4$ gcd7 $\triangle::$ His3 & pSD452/pSD469 & $\begin{array}{l}\text { pAH056 CEN6/ARS4 URA3 sui2-2 } \\
\text { pAH005 CEN6/ARS4 LEU2 gcd7-2 }\end{array}$ & This study \\
\hline sSD060 & MATa his $3 \Delta 1$ leu2 $\triangle 0$ met $15 \Delta 0$ ura $3 \triangle 0$ sui2 $\triangle:: K a n M X 4$ gcd $7 \Delta::$ His3 & pSD444/pSD474 & $\begin{array}{l}\text { pAH056 CEN6/ARS4 URA3 SUI2 } \\
\text { pAH005 CEN6/ARS4 LEU2 GCD7 }\end{array}$ & This study \\
\hline sSD061 & MATa his $3 \Delta 1$ leu $2 \Delta 0$ met $15 \Delta 0$ ura $3 \Delta 0$ & pSD442 & pAH056 CEN6/ARS4 URA3 GCD1 & This study \\
\hline sSD062 & MATa his $3 \Delta 1$ leu $2 \Delta 0$ met $15 \Delta 0$ ura $3 \Delta 0$ & pSD450 & pAH056 CEN6/ARS4 URA3 gcd1-1 & This study \\
\hline sSD063 & MATa his $3 \Delta 1$ leu $2 \Delta 0$ met $15 \Delta 0$ ura $3 \Delta 0$ & pSD444 & pAH056 CEN6/ARS4 URA3 SUI2 & This study \\
\hline sSD064 & MATa his $3 \Delta 1$ leu $2 \Delta 0$ met $15 \Delta 0$ ura $3 \Delta 0$ & pSD452 & pAH056 CEN6/ARS4 URA3 sui2-2 & This study \\
\hline
\end{tabular}

Highlighted in bold are mutant alleles identified in the evolved strains

a Dept. of Bioengineering, University of California Berkeley

PCR amplified to give a product with sequences homologous ( $40 \mathrm{bp}$ ) to those flanking the GCD7 gene. Similarly, PCR products with the kanamycin/G418 resistance (KanMX) marker from BY4741 $\Delta$ tpo1 (MATa his3 $\Delta 1$ leu $2 \Delta 0$ met $15 \Delta 0$ ura3 $\Delta 0$ tpo1 $\triangle:: k a n M X 4)$ were generated to delete GCD1 and SUI2. Strains BY4741[pSD469] and BY4741[pSD474] were transformed with the GCD7 deletion cassette to obtain sSD040 and sSD050, respectively. $\mathrm{LEU}^{+} \mathrm{HIS}^{+}$transformants were selected on SD medium lacking leucine and histidine. Strains BY4741[pSD450] and BY4741[pSD442] were transformed with the GCD1 cassette to obtain sSD029 and sSD053, respectively, and strains BY4741[pSD444] and BY4741[pSD452] were transformed with the SUI2 cassette to obtain sSD055 and sSD055, respectively. $U R A^{+} K a n M X^{+}$transformants were selected on SD medium lacking uracil and supplemented with $200 \mathrm{mg} / \mathrm{l} \mathrm{Geneticin} \mathrm{(G418} \mathrm{sulfate).} \mathrm{Finally,}$ BY4741[pSD452/pSD469] and BY4741[pSD444/pSD474] were transformed with the GCD7 and SUI2 deletion cassettes to obtain sSD058 and sSD060, respectively. These transformations were plated on SD medium lacking uracil, leucine and histidine, and uracil, leucine with Geneticin, respectively. Gene deletions were confirmed by diagnostic PCR across the junctions of the expected integration site on genomic DNA templates isolated from these strains. Primer pairs were GCD1-F/KanMXdiagnostic- $\mathrm{R}$ and GCD1-R/KanMX-diagnostic-F, for the GCD1 gene deletion, SUI2-F/KanMX-diagnostic-R and SUI2-R/KanMX-diagnostic-F, for the SUI2 deletion, and GCD7-HIS3-F/HIS3-diagnostic-R for HIS3, for the GCD7 deletion (Additional file 1: Table S1).

Single amino-acid substitution libraries at positions D85 in Gcd1p and D77 Sui2p D77 were generated by PCR in which the codon for the substituted amino-acid was randomized by degenerate NNN and NNK primers (Additional file 1: Table S1), respectively, where $\mathrm{N}$ is an equimolar mixture of all four nucleotides and $\mathrm{K}$ is an equimolar mixture of bases G and T. GCD1 and SUI2 genes were amplified as two products using two partially overlapping mutagenic primers and two primers outside the coding sequence, and pSD442 and pSD444 plasmid DNA as templates, respectively. The mutant gene sequences were introduced into the appropriate destination vector by HR in yeast. The destination vectors for Gcd1p and Sui2p mutants were constructed by HR from pSD442 (ClaI digested) and pSD444 (BglII digested) by replacing the GCD1 and SUI2 coding sequence with the KanMX marker. For each library of single amino-acid substitution yeast mutants, 96 colonies were sequenced and analyzed.

\section{Adaptive laboratory evolution}

ALE was performed by a serial batch transfer procedure and by batch fermentation in a chemostat in the presence 
of inhibitory concentrations of $n$-hexanol as a selective pressure. In the serial batch procedure, a single aerobic culture of strain BY4741 was initiated in $10 \mathrm{ml}$ YPD supplemented with $0.12 \%(\mathrm{v} / \mathrm{v}) n$-hexanol in $50 \mathrm{ml}$ screw-cap tubes and grown at $30{ }^{\circ} \mathrm{C}$ and $200 \mathrm{rpm}$. This culture was passaged daily in fresh YPD with $0.12 \% n$-hexanol. After 30 daily passages, a diluted aliquot of the final culture was plated for single colonies on YPD. Yeast populations derived from a number of colonies were tested for their $n$-hexanol tolerance (see "Tolerance testing" below), as compared to the parental strain, and the best performing strain was designated sSD003.

For batch fermentation, BY4741 was cultivated in a 0.51 chemostat containing 0.51 YPD. Fresh medium was fed at a constant flow-rate in the range $0.25-0.5 \mathrm{ml} / \mathrm{min}$ so that the $\mathrm{OD}_{600}$ of the culture was maintained in the range 0.5-2.0 AU. The initial $n$-hexanol concentration was $0.1 \%$, but as the cell density increased, the $n$-hexanol concentration was steadily increased to $0.3 \%$. The chemostat had no air inlet or outlet, so the culture was grown under microaerobic conditions, mimicking those found in large-scale fermentation tanks. The chemostat was kept in a $30{ }^{\circ} \mathrm{C}$ room and the culture mixed with a medium-speed stir bar. At the end of the 30 day fermentation, several strains derived from the final culture were tested for $n$-hexanol tolerance, and the best performing strain was designated sSD006.

Strains sSD003 and sSD006 were subjected individually to a second 30-day round of adaptive evolution by serial batch transfer in tubes. The starting $n$-hexanol concentration was $0.12 \%$, and this was increased gradually to $0.2 \%$ over the 30 -day period. After tolerance testing of several strains derived from sSD003, we identified strain sSD019 which showed significantly increased $n$-hexanol tolerance compared to the parental strain. Similarly, a significantly more $n$-hexanol-tolerant strain, sSD021, was obtained from the adaptive evolution of sSD006.

\section{Tolerance testing}

Strains were evaluated for tolerance to medium-chain alcohols by determining growth curves. Overnight cultures were diluted to an $\mathrm{OD}_{600}$ of $0.3 \mathrm{AU}$ in $5 \mathrm{ml} \mathrm{YPD}$ and then mixed with $5 \mathrm{ml}$ YPD containing two times the desired final concentration of the test alcohol (expressed as $\mathrm{v} / \mathrm{v}$ ). Cultures were grown in closed $50 \mathrm{ml}$ screw-cap tubes (to prevent the evaporation of test alcohols) at $30{ }^{\circ} \mathrm{C}$ and $280 \mathrm{rpm}$. The $\mathrm{OD}_{600}$ of the cultures was measured every $2 \mathrm{~h}$ in triplicate with a Genesys 20 spectrophotometer. The average specific growth rate $\left(\mu_{\text {avg }}\right)$ for each culture was calculated for the time period between 2 and $10 \mathrm{~h}$ (except where otherwise indicated) using the following equation: $\mu=\left(\ln X_{2}-\ln X_{1}\right) /\left(t_{1}-t_{2}\right)$, where $X_{2}$ and $X_{1}$ are the cell density at time $t_{2}$ and $t_{1}$.

\section{Genomic sequencing}

Genomic DNA was isolated from the evolved strains with the YeaStar ${ }^{\mathrm{TM}}$ genomic DNA kit. Barcode-indexed genomic DNA sequencing libraries were generated from $1 \mu \mathrm{g}$ of DNA each. The DNA samples were sheared on a Diagenode Bioruptor NGS sonicator (Diagenode, Liege, Belgium) to an average length of $300 \mathrm{bp}$. The libraries were prepared with the TruSeq Library Prep Kit (Illumina, San Diego, CA) following the instructions of the manufacturer. Libraries were analyzed with a Bioanalyzer 2100 instrument (Agilent, Santa Clara, CA), quantified by fluorometry on a Qubit instrument (LifeTechnologies, Carlsbad, CA) and pooled in equimolar ratios. The library pool was quantified by qPCR with a Kapa Library Quant RT-qPCR kit (Kapa, Cape Town, South Africa). 8.2 pM of library was loaded and ran on 1 lane of an Illumina HiSeq 2000 (Illumina, San Diego, $\mathrm{CA}$ ). The raw reads were trimmed to remove sequencing adaptors as well as quality trimmed using Trimmomatic v0.36 [29] to have a minimum quality of 14 in a sliding window of 4 . Paired reads that are longer than 36 bp were kept for further analysis. Mapping to the $S$. cerevisiae S288C genome (R64-2-1) obtained from the Saccharomyces Genome Database was done using the Burrows Wheel Aligner v0.7.12 MEM algorithm [30]. The mapped reads were then sorted and duplicated reads were marked using Picard v2.9.2. Variant calling was done using freebayes v1.1.0.46 [31] with the ploidy option set to 1 . Variants with quality less than 20 were then filtered out using vcflib v1.0.0. The variants found in parental strain were removed from those found in each evolved strain with bedtools v2.26.0 [32]. Annotation was done using both SnpEff v4.3 [33] and Bcftools v1.6. The SnpEff output was used to decipher the potential effects of the mutations. Raw reads have been deposited in the NCBI Sequence Read Archive under the accession number PRJNA417511.

\section{Polysome profiling}

Polysome analysis was performed as previously described [34]. Briefly, strains were grown in $250 \mathrm{ml}$ of YPD to an $\mathrm{OD}_{600}$ of $\sim 1 \mathrm{AU}$. The test alcohol was then added and the culture incubated for a further $30 \mathrm{~min}$. Cycloheximide $(100 \mu \mathrm{g} / \mathrm{ml})$ was added to each culture for $5 \mathrm{~min}$ to arrest translation. Cells were harvested, washed twice, and then lysed with glass beads in ice-cold breaking buffer (20 mM Tris/ $\mathrm{HCl} \mathrm{pH} \mathrm{7.4,} 50 \mathrm{mM} \mathrm{KCl}, 10 \mathrm{mM} \mathrm{MgCl}$, $1 \mathrm{mM}$ DTT, $100 \mu \mathrm{g} / \mathrm{ml}$ cycloheximide, $50 \mathrm{U} / \mathrm{ml}$ SuperRNasein, and protease inhibitors), followed by centrifugation at $13,000 \times g$ for $20 \mathrm{~min}$ at $4{ }^{\circ} \mathrm{C} .20 \mathrm{~A}_{260 \mathrm{~nm}}$ units $(800 \mu \mathrm{g}$ of RNA) of each supernatant was fractionated on $10-50 \%$ sucrose gradients for $3 \mathrm{~h}$ at $35,000 \mathrm{rpm}$ and $4{ }^{\circ} \mathrm{C}$ using a SW40-Ti rotor in a Optima L-90 K 
ultracentrifuge (Beckman Coulter). Polysome profiles were obtained by monitoring the RNA absorbance at $254 \mathrm{~nm}$ along the gradient using a BR-188 Density Gradient Fractionation System, and recording the output from the UA-6 Detector with programmable RS-232 software (Brandel, Gaithersburg, Maryland). Polysome-to-monosome $(\mathrm{P} / \mathrm{M})$ ratios were determined by calculating the area under the curve corresponding to the polyribosome peaks (more than two ribosomes) divided by the area under the curve for the monosome (80S) peak.

\section{Results}

\section{Adaptive laboratory evolution of medium-chain alcohol} tolerance in S. cerevisiae

To select for spontaneous mutants with greater tolerance to $n$-hexanol, two independent evolutionary lineages of wild-type BY4741 were established in the presence of the alcohol. The first of these was grown aerobically in $50 \mathrm{ml}$ tubes in the presence of $0.12 \% n$-hexanol and was serially passaged for 30 days (serial batch transfer). This concentration was chosen based on our observations that it resulted in severely limited growth. Several strains were then isolated from this population as single colonies on plates and tested for $n$-hexanol tolerance. Tolerance testing was performed by determining growth curves with measurements made every $2 \mathrm{~h}$. Strains were grown in closed $50 \mathrm{ml}$ screw-cap tubes to prevent evaporation of $n$-hexanol, and the average specific growth rates $\left(\mu_{\text {avg }}\right)$ were calculated for the time period between 0 and $10 \mathrm{~h}$ to ensure that measurements were made on aerobically growing yeast (see representative growth curves for evolved strains in Additional file 1: Figure S1). For the best performing strain, sSD003, $\mu_{\text {avg }}$ was increased $\sim$ sixfold compared to the parental strain $(P<0.001)$ in the presence of $0.15 \% n$-hexanol (Fig. 1a). sSD003 was then subjected to another 30-day round of adaptive evolution by serial batch transfer with an $n$-hexanol concentration of $0.12 \%$ that was increased gradually to $0.21 \%$. The best performing strain of those tested from the final culture was designated sSD019 and it grew $\sim$ sevenfold faster than the wild-type strain $(P<0.001)$ in $0.15 \% n$-hexanol (Fig. 1a).

The second evolutionary lineage was grown in a microaerobic chemostat for 30 days with an initial $n$-hexanol concentration of $0.1 \%$ that was increased steadily with increasing cell density to a final concentration of $0.3 \%$. Strain sSD006 was isolated from the final culture that grew $\sim$ threefold faster than the parental strain $(P<0.001)$. This lineage was further extended by 30 days of serial batch transfer in tubes with $0.12-0.21 \% n$-hexanol. This allowed the isolation of sSD021 which exhibited a $\sim 5$-fold increased $\mu_{\text {avg }}$ compared to wild-type BY4741 $(P<0.001)$ (Fig. 1a). In the absence of $n$-hexanol,

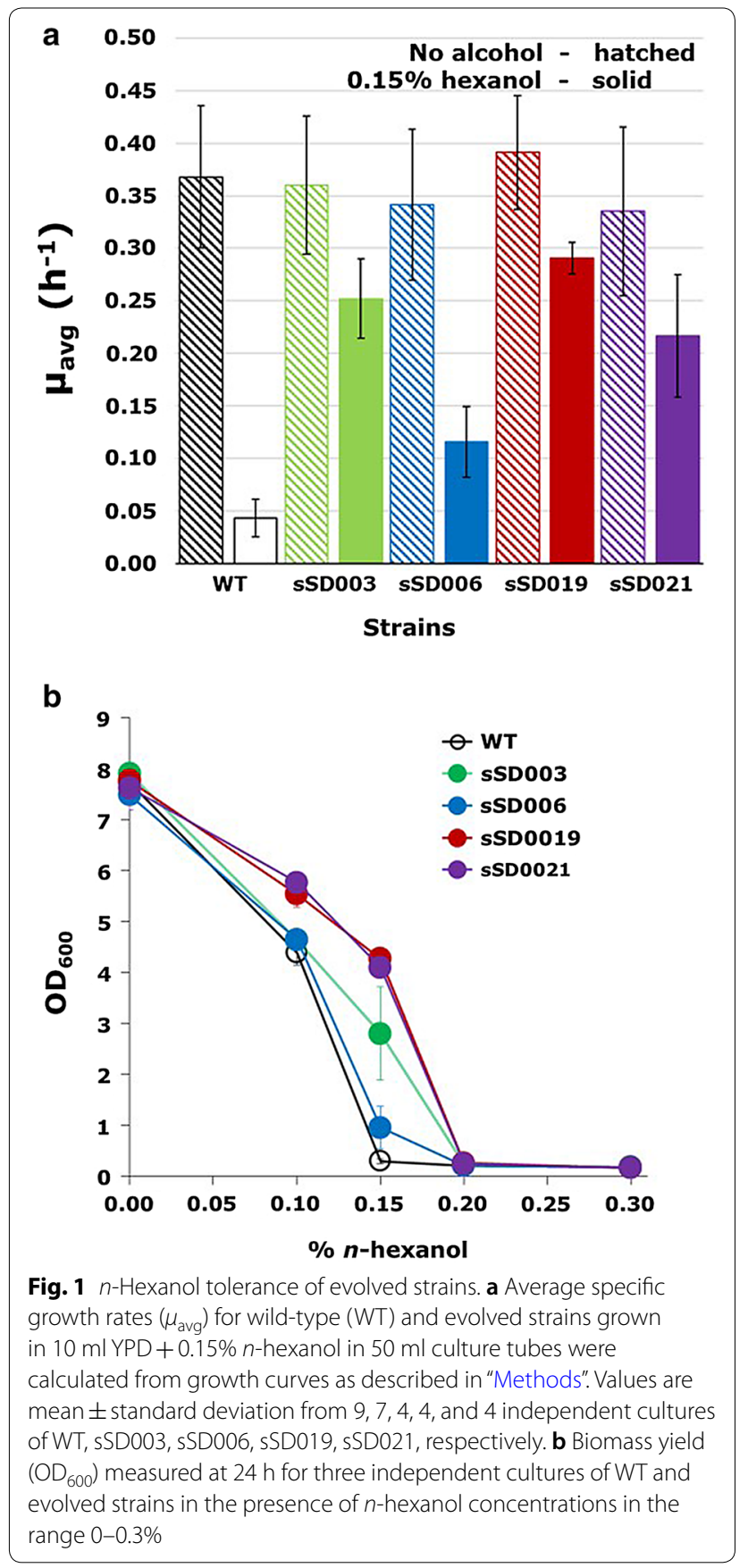

the evolved mutants grew as well as the wild-type strain $\left[0.37 \pm 0.07 \mathrm{~h}^{-1}(n=21), 0.36 \pm 0.07 \mathrm{~h}^{-1}(n=18)\right.$, $0.34 \pm 0.07 \mathrm{~h}^{-1} \quad(n=12), 0.39 \pm 0.05 \mathrm{~h}^{-1} \quad(n=15)$, and $0.34 \pm 0.08 \mathrm{~h}^{-1}(n=14)$ for WT, sSD003, sSD006, sSD019 and sSD021, respectively].

To identify the upper range of $n$-hexanol tolerance for the evolved strains, we measured the biomass $\left(\mathrm{OD}_{600}\right)$ of cultures of the WT and evolved strains following $24 \mathrm{~h}$ growth in the presence of $n$-hexanol concentrations in the range $0-0.3 \%$ (Fig. 1b). At an $n$-hexanol 
concentration of $0.2 \%$, the $\mathrm{OD}_{600}$ values for sSD003 and sSD006 were not significantly different from that for the WT strain $[0.234 \pm 0.013(n=3), 0.211 \pm 0.009(n=3)$, and $0.202 \pm 0.018(n=3)$, respectively]. In contrast, the $\mathrm{OD}_{600}$ values for the more evolved strains sSD019 and sSD021 were significantly greater than that for the WT strain $(P<0.05)[0.258 \pm 0.023(n=3)$ and $0.243 \pm 0.018$ $(n=3)$, respectively], demonstrating that they can grow at this concentration of $n$-hexanol.

To investigate whether the evolved $n$-hexanol-tolerant strains were also more tolerant to other higher alcohols, we tested their growth in the presence of toxic concentrations of $n$-propanol ( $\mathrm{PrOH}), n$-butanol $(\mathrm{BuOH})$, isobutanol $(\mathrm{iBuOH})$, amyl alcohol $(\mathrm{AOH})$, isoamyl alcohol (iAOH), n-pentanol (PtOH), $n$-heptanol $(\mathrm{HpOH})$, and 3-octanol $(\mathrm{OcOH})$ (Table 2). All alcohols tested inhibited the growth of the WT strain but to different extents at the concentrations used. The strains SSD003 and sSD019 in one lineage were significantly more tolerant than the wild-type strain to $3 \% n$-propanol, growing three- to fourfold faster $(P<0.01)$. Interestingly, sSD021 in the other lineage was significantly less tolerant to $n$-propanol $(P<0.05)$. sSD003 was also more tolerant to $1.2 \%$ $n$-butanol (1.3-fold), whereas sSD021 was less tolerant $(P<0.001)$. The more evolved strains, sSD019 and sSD021, were more tolerant to $1.2 \%$ isobutanol, growing $\sim$ 1.4-fold faster than the parental strain $(P<0.05)$. Moreover, the evolved strains showed improved tolerance to $0.6 \%$ amyl alcohol growing $\sim 2$ to 3 -fold faster, and sSD019 and sSD021 had improved tolerance to $0.4 \%$ isoamyl alcohol growing about $20 \%$ faster $(P<0.05)$. A pattern of improved tolerance of the evolved strains similar to that seen in $n$-hexanol (Fig. 1 ), namely sSD019 $\cong$ sSD021 $>$ sSD003 $>$ sSD006, was observed with the longer-chained alcohols $0.5 \% n$-pentanol, $0.05 \% n$-heptanol, and 0.05\% 3-octanol.

\section{Identification of mutations affecting $n$-hexanol tolerance by whole-genome sequencing}

To identify genetic targets that can be exploited to alleviate medium-chain alcohol toxicity in S. cerevisiae, genomic DNA was isolated from the evolved strains and sequenced to obtain the whole-genome. Mutations were identified by comparison of each sequenced genome to that of the parental strain, which was also sequenced. All of the mutations for each strain are listed in Table 3. Strikingly, $n$-hexanol-tolerant strains in both evolutionary lineages had mutations in translation initiation proteins. These proteins were Gcd1p, the $\gamma$ subunit of the translation initiation factor eIF2B, Gcd7p, the $\beta$ subunit of eIF2B, and Sui2p, the $\alpha$ subunit of the translation initiation factor eIF2 (Table 4). The strains SSD003 and sSD019 in one lineage had a D85E mutation in Gcd1p (here designated as the $\operatorname{gcd} 1-1$ allele), whereas sSD006 and sSD021 in the other lineage had an R56C mutation in Gcd7p $(g c d 7-2)$. Strain sSD021 had a second translation initiation protein mutation D77Y in Sui2p (sui22). Also of interest is the observation that in the more evolved strains in the two lineages, sSD019 and sSD021, mutations were found in the plasma membrane $A B C$ transporter Pdr5p, one of the major multidrug exporters of S. cerevisiae. Strain SSD019 was found to harbor a Q446* mutation ( $p d r 5-1)$ that would result in a truncated Pdr5p (tPdr5 $\left.\mathrm{p}^{1-446}\right)$, and sSD021 had Pdr5p with a G925A mutation (pdr5-2). Because the other mutations predominantly identified in the more evolved strains differed between the lineages, we assumed that they were passenger mutations that arose from genetic drift, and decided to focus our attention on the translation initiation protein mutations and the role of Pdr5p only.

To investigate whether the mutations in the translation initiation proteins contribute to the $n$-hexanol tolerant phenotype, we reversed engineered the $\operatorname{gcd} 1-1, \operatorname{gcd} 7-2$,

Table 2 Medium-chain alcohol tolerance of evolved strains

\begin{tabular}{|c|c|c|c|c|c|c|c|c|c|}
\hline \multirow[t]{3}{*}{ Strains } & \multicolumn{9}{|l|}{$\mu_{\mathrm{avg}}\left(\mathrm{h}^{-1}\right)^{*}$} \\
\hline & \multicolumn{9}{|c|}{ Alcohol $(\%, v / v)$} \\
\hline & $\operatorname{PrOH}(3)^{a}$ & $\mathrm{BuOH}(1.2)^{\mathrm{a}}$ & iBuOH (1.2) & $\mathrm{AOH}(0.6)$ & iAOH (0.4) & $\operatorname{PtOH}(0.6)^{\mathrm{a}}$ & $\mathrm{HxOH}(0.15)^{a}$ & $\mathrm{HpOH}(0.05)^{\mathrm{a}}$ & $\mathrm{OcOH}(0.05)^{\mathrm{a}}$ \\
\hline WT & $0.042 \pm 0.004$ & $0.144 \pm 0.014$ & $0.195 \pm 0.032$ & $0.044 \pm 0.013$ & $0.264 \pm 0.027$ & $0 \pm 0.010$ & $0.043 \pm 0.018$ & $0 \pm 0.009$ & $0 \pm 0.008$ \\
\hline sSD003 & $0.172 \pm 0.030^{\#}$ & $0.170 \pm 0.005^{*}$ & $0.202 \pm 0.020$ & $0.096 \pm 0.026^{*}$ & $0.294 \pm 0.023$ & $0.133 \pm 0.034^{\#}$ & $0.252 \pm 0.038^{\dagger}$ & $0.051 \pm 0.026^{*}$ & $0.092 \pm 0.009^{\dagger}$ \\
\hline sSD006 & $0.038 \pm 0.004$ & $0.134 \pm 0.021$ & $0.211 \pm 0.027$ & $0.071 \pm 0.024$ & $0.285 \pm 0.024$ & $0.145 \pm 0.103$ & $0.115 \pm 0.034^{\dagger}$ & $0 \pm 0.012$ & $0.033 \pm 0.021 *$ \\
\hline sSD019 & $0.129 \pm 0.022^{\#}$ & $0.140 \pm 0.032$ & $0.267 \pm 0.017^{*}$ & $0.128 \pm 0.019^{\#}$ & $0.318 \pm 0.006^{*}$ & $0.161 \pm 0.090^{*}$ & $0.291 \pm 0.015^{\dagger}$ & $0.290 \pm 0.028^{\dagger}$ & $0.274 \pm 0.063^{*}$ \\
\hline sSD021 & $0.029 \pm 0.042^{*}$ & $0.063 \pm 0.001^{\dagger}$ & $0.264 \pm 0.022^{*}$ & $0.139 \pm 0.028^{\#}$ & $0.311 \pm 0.017$ & $0.260 \pm 0.072^{\#}$ & $0.217 \pm 0.058^{\dagger}$ & $0.247 \pm 0.007^{\dagger}$ & $0.323 \pm 0.020^{\dagger}$ \\
\hline
\end{tabular}

a Differences in growth between strains in these alcohols was only apparent at time intervals beyond the 2-10-h period used to calculate $\mu_{\text {avg }}$ for iBuOH, $\mathrm{AOH}$, iAOH, and $\mathrm{HxOH}$. The $\mu_{\text {avg }}$ in these alcohols was calculated for time periods when the cells were in the exponential growth phase. At these longer time interval, in tightly capped tubes, growth may not have been under fully aerobic conditions. Representative growth curves for these alcohols are given in Additional file 1: Figure S2 $(\mathrm{PtOH})$ and Additional file 1: Figure $\mathrm{S} 3(\mathrm{HpOH})$

* Values are mean \pm standard deviation evaluated from three biological replicates for all samples except $\mathrm{HxOH}$, for which there were nine biological replicates for WT, seven for sSD003, and four each for the other three strains. Statistical significance was determined by an unpaired two-tailed Student's $t$ test; significant differences from the WT are indicated as follows: ${ }^{\dagger} P \leq 0.001 ;{ }^{\#} P \leq 0.01 ;{ }^{*} P \leq 0.05$ 
Table 3 Summary of mutations in evolved strains identified by whole-genome resequencing

\begin{tabular}{|c|c|c|c|c|}
\hline Strain & Mutation & Gene & Protein function ${ }^{\mathbf{b}}$ & Mutant allele \\
\hline \multirow[t]{2}{*}{ sSD003 } & D85E & GCD1 & Gamma subunit of the translation initiation factor elF2B & $\operatorname{gcd} 1-1$ \\
\hline & G338C & CIT2 & Citrate synthase, peroxisomal isozyme involved in glyoxylate cycle & cit2-1 \\
\hline sSD006 & R56C & GCD7 & Beta subunit of the translation initiation factor elF2B & $\operatorname{gcd} 7-2$ \\
\hline \multirow[t]{7}{*}{ sSD019 } & D85E & GCD1 & Gamma subunit of the translation initiation factor elF2B & $\operatorname{gcd} 1-1$ \\
\hline & G338C & CIT2 & Citrate synthase, peroxisomal isozyme involved in glyoxylate cycle & cit2-1 \\
\hline & Q446 ${ }^{\mathrm{a}}$ & PDR5 & Plasma membrane ATP-binding cassette (ABC) transporter & $p d r 5-1$ \\
\hline & G135V & UBP13 & Ubiquitin-specific protease & ubp 13-1 \\
\hline & S234C & LSB6 & Type II phosphatidylinositol 4-kinase & Isb6-1 \\
\hline & A662G & NST1 & Protein of unknown function; mediates sensitivity to salt stress & nst1-1 \\
\hline & Silent & $\operatorname{cox} 1$ & Subunit I of cytochrome c oxidase (Complex IV) & $\cos 1-1$ \\
\hline \multirow[t]{4}{*}{ sSD021 } & R56C & GCD7 & Beta subunit of the translation initiation factor elF2B & $\operatorname{gcd} 7-2$ \\
\hline & D77Y & SUI2 & Alpha subunit of the translation initiation factor elF2 & suiz-2 \\
\hline & G925A & PDR5 & Plasma membrane ATP-binding cassette (ABC) transporter & $p d r 5-2$ \\
\hline & A529E & SEY1 & Dynamin-like GTPase that mediates homotypic ER fusion & $\operatorname{sey} 1-2$ \\
\hline
\end{tabular}

a Denotes the introduction of an early stop codon

b Protein function taken from the Saccharomyces genome database website http://www.yeastgenome.org

Table 4 Subunits of eIF2 and elF2B

\begin{tabular}{llll}
\hline & Subunit & Protein & Function \\
\hline elF2 & $a$ & Sui2p & Regulation of elF2 activity \\
& $\beta$ & Sui3p & tRNA binding \\
& $\gamma$ & Gcd1 1p & GTP binding \\
elF2B & $a$ & Gcn3p & Regulatory subunit; elF2B regulation \\
& $\beta$ & Gcd7p & Regulatory subunit, role in elF2 binding \\
& $\gamma$ & Gcd1p & Catalytic, non-active, role in elF2 binding \\
& $\delta$ & Gcd2p & Regulatory subunit; elF2B formation \\
& $\varepsilon$ & Gcd6p & GDP to GTP exchange \\
\hline
\end{tabular}

and sui2-2 alleles in BY4741. As the GCD1, GCD7, and SUI2 genes are essential for viability, the mutant alleles (or the wild-type alleles as controls) were first introduced individually (or in combination as indicated) on low-copy plasmids into BY4741, and then the wild-type genes in the genome of transformed strains corresponding to those on the plasmids were deleted. The $n$-hexanol tolerance of the resulting strains (Table 1) was tested and compared to the parental strain and the relevant evolved strain (Fig. 2). The introduction of $\operatorname{gcd} 1-1$, but not $G C D 1^{+}$, into BY4741 $g c d 1 \Delta$ resulted in a strain (sSD029) that was significantly more tolerant to $0.15 \% n$-hexanol than the wild-type strain $(P<0.05)$ (Fig. $2 b)$, though less tolerant than SSD003, the evolved strain with the $\operatorname{gcd} 1$ 1 allele $(P<0.05)$. These results confirm that $g c d 1-1$ confers $n$-hexanol tolerance in BY4741. Likewise, the strain sSD056 with the sui2-2 allele, but not that carrying the SUI $^{+}$allele (sSD055), was significantly more tolerant to $0.15 \% n$-hexanol than the wild-type strain $(P<0.05)$ (Fig. 2f), and exhibited a tolerance level comparable to sSD021, the evolved strain with the sui2-2, $g c d 7-2$, and pdr5-2 alleles. A strain sSD058 carrying both sui2-2 and $g c d 7-2$ exhibited an $n$-hexanol tolerance (Fig. 2h) similar to that seen with sSD056 which had only sui2-2, suggesting that the tolerance of these strains is largely accounted for by the presence of the sui2-2 allele. These results confirm that sui2-2 confers $n$-hexanol tolerance in BY4741. In contrast to the results with $g c d 1-1$ and sui2-2, the

\footnotetext{
(See figure on next page.)

Fig. 2 Reverse engineering of $g c d 1-1, g c d 7-2$, and sui2-2 in $\operatorname{gcd} 1 \mathrm{D}, \mathrm{gcd} 7 \mathrm{D}$, and sui2D strains, respectively. For the gcd1 allelic variants, strains sSD003, sSD053, and sSD029 were grown in $10 \mathrm{ml} \mathrm{YPD} \mathrm{in} \mathrm{the} \mathrm{absence}(\mathbf{a})$ or presence $(\mathbf{b})$ of $0.15 \% \mathrm{n}$-hexanol, and their $\mu_{\text {avg }}$ values are shown. For the gcd7 allelic variants, strains SSD006, SSD054, and sSD040 were grown in $10 \mathrm{ml} \mathrm{YPD} \mathrm{in} \mathrm{the} \mathrm{absence} \mathrm{(c)} \mathrm{or} \mathrm{presence} \mathrm{(d)} \mathrm{of} 0.15 \% \mathrm{n}$-hexanol, and their $\mu_{\text {avg }}$ values are shown. For the sui2 allelic variants, strains sSD021, sSD055, and sSD056 were grown in $10 \mathrm{ml} \mathrm{YPD} \mathrm{in} \mathrm{the} \mathrm{absence} \mathrm{(e)} \mathrm{or} \mathrm{presence} \mathrm{(f)} \mathrm{of}$ $0.15 \%$ nhexanol, and their $\mu_{\text {avg }}$ values are shown. For the gcd 1 allelic variants, strains sSD021, sSD060, and sSD058 were grown in $10 \mathrm{ml} \mathrm{YPD} \mathrm{in} \mathrm{the}$ absence $(\mathbf{g})$ or presence $(\mathbf{h})$ of $0.15 \%$-hexanol, and their $\mu_{\text {avg }}$ values are shown. The $\mu^{\text {avg }}$ values for WT, sSD003, sSD006 and sSD021 in the absence of alcohol are also given in the text and they are shown here for comparison with relevant reverse-engineered strains. The values for WT, sSD003, sSD006 and sSD021 in the presence of 0.15\% n-hexanol are the same as in Fig. 1 and are given for comparison. Identical data for WT are shown in multiple plots for comparison and data for strain sSD021 is shown in both $\mathbf{e}, \mathbf{f}$ and $\mathbf{g}, \mathbf{h}$ for comparison. Values are means \pm standard deviation from three independent cultures
} 

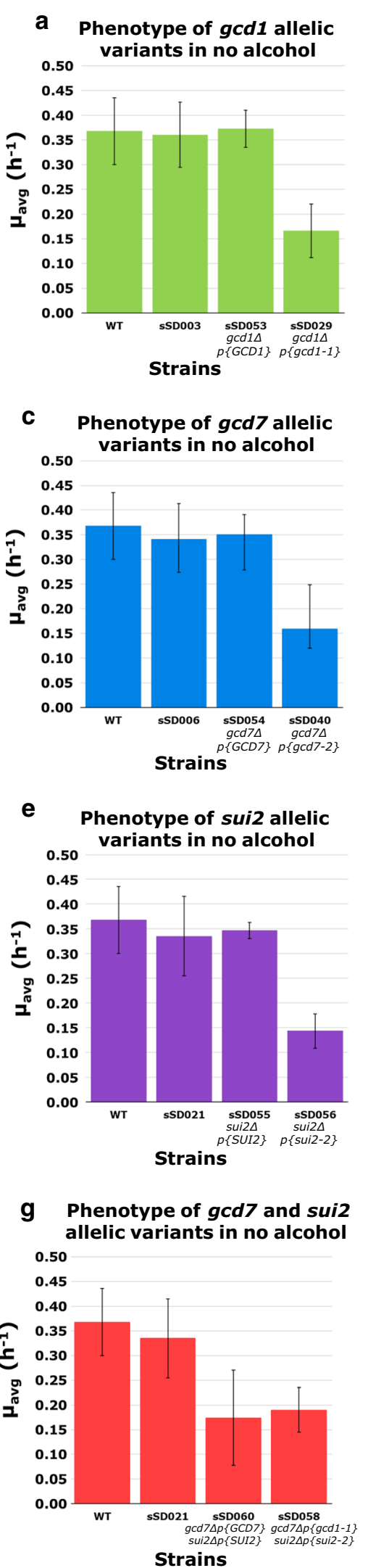
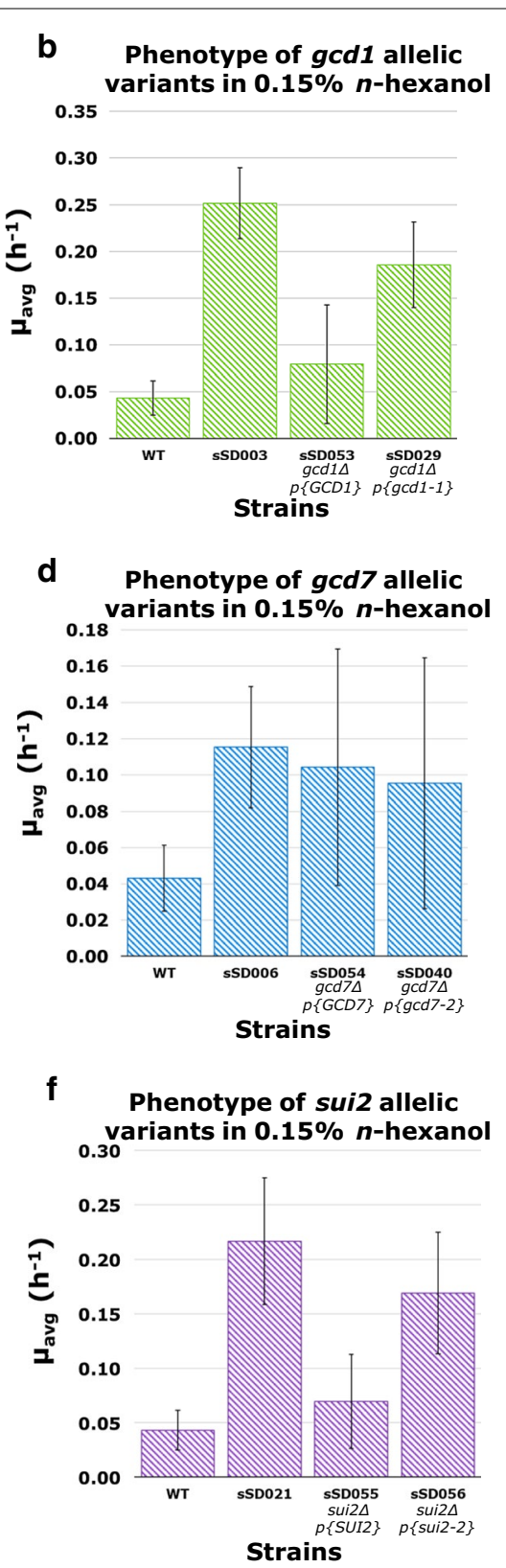
h Phenotype of $\mathbf{g c d} 7$ and sui2 allelic variants in $0.15 \% n$-hexanol 0.30

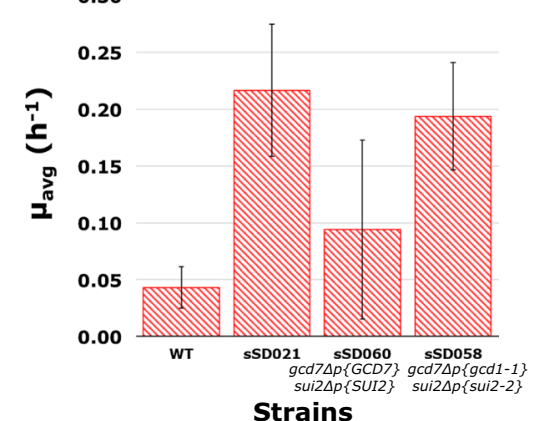


strain with the reverse-engineered mutant $g c d 7-2$ allele (sSD040), a reconstruction of sSD006, was not significantly more tolerant to $0.15 \% n$-hexanol than the wildtype strain (Fig. 2d).

Interestingly, although plasmid-borne $G C D 1^{+}$fully complemented the $\operatorname{gcd} 1 \Delta$ in BY4741 in the absence of alcohol, as evidenced by strain sSD053 having the same growth rate as wild-type BY4741 (Fig. 2a), plasmid-borne $g c d 1$-1 did not [sSD029 grew $\sim 2$-fold slower than WT in the absence of alcohol $(P<0.05)]$, suggesting that the expression level of $g c d 1-1$ achieved with the plasmid may be lower than that from the chromosome in sSD003. This may account for the failure of plasmid-borne $g c d 1-1$ to fully restore the degree of $n$-hexanol tolerance seen with sSD003. A lack of full complementation of gene deletions was also observed with the other mutant alleles [ $g c d 7-2$ (Fig. 2c), sui2-2 (Fig. 2e), and $g c d 7-2$ and sui2-2 (Fig. 2g)]. Moreover, in strain sSD060 (Fig. 2g), full complementation was not achieved with plasmid-borne $G C D 7^{+}$and $S U I 2^{+}$, perhaps reflecting the additional metabolic burden of carrying and replicating two plasmids.

Unpublished work reported in a patent by Tina K. Van Dyk (Butamax ${ }^{\mathrm{Tm}}$ Advanced Biofuels Llc) [35] demonstrated that the haploid strain BY4741

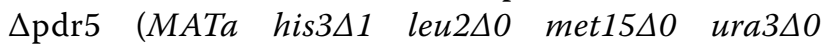
pdr5 $4:$ :kanMX4) [36] showed improved tolerance to isobutanol as compared to the parental strain (eightfold increase in $24 \mathrm{~h}$ biomass yield in the presence of $1 \%(\mathrm{w} / \mathrm{v})$ isobutanol). A homozygous diploid $\Delta \mathrm{pdr} 5$ strain showed improvements in tolerance to $n$-butanol [1.4-fold increase in $24 \mathrm{~h}$ biomass yield in the presence of $0.625 \%(\mathrm{w} / \mathrm{v}) n$-butanol]. Pdr5p is known to have a high basal ATPase activity and to be among the major consumers of cellular energy [37], and thus the improvements in isobutanol and butanol tolerance observed for the $\Delta \mathrm{pdr} 5$ strains likely reflects improved fitness due to loss of the protein. The Q446* mutation in Pdr5p encoding a truncated version of the protein $\left(\operatorname{tPdr} 5 \mathrm{p}^{1-446}\right)$ would certainly abolish transporter activity as only the $\mathrm{N}$-terminal nucleotide-binding domain and not the two transmembrane domains would be expressed [37]. To investigate whether the loss of Pdr5p could make a contribution to the $n$-hexanol tolerance phenotype, we compared the $n$-hexanol tolerance of BY4741 $\Delta$ pdr5 with the WT strain. Surprisingly, the $\Delta$ pdr 5 strain did not show improved $n$-hexanol tolerance [Additional file 1: Figure S4; $0.055 \pm 0.003 \mathrm{~h}^{-1}(n=3)$ and $0.057 \pm 0.005 \mathrm{~h}^{-1}(n=3)$, respectively]. This result does not rule out the possibility that the evolved tPdr5p $\mathrm{p}^{1-446}$ strain, sSD019, (and/or the sSD021 strain with the G925A mutation in $\operatorname{Pdr} 5 \mathrm{p}$ ) has a gain-of-function mutation that only becomes important when $n$-hexanol toxicity-alleviating mutations are already present in translation initiation proteins.

\section{Mutations in Gcd1p and Sui2p relieve inhibition of translational activity by higher alcohols}

To probe the mechanism by which mutations in Gcd1p and Sui2p cause improved $n$-hexanol tolerance of yeast cells, we examined the translational activity of cells in the presence and absence of $n$-hexanol by polysome profiling. Translational activity is usually expressed as the polysome-to-monosome $(\mathrm{P} / \mathrm{M})$ ratio, which, in principle, decreases when translation initiation is defective and increases with elongation defects. In the former case, there is a reduction in the number of ribosomes translating a given mRNA, and hence a reduction in the abundance of polysomes together with a concomitant increase in the amount of free 80S ribosomes [34]. Figure $3 \mathrm{a}, \mathrm{c}$ shows that the $\mathrm{P} / \mathrm{M}$ ratio for wild-type cells is dramatically reduced following a 30-min incubation with $0.15 \% n$-hexanol $(\sim 14$-fold $)(P<0.05)$. Consistent with the hypothesis that translation initiation is inhibited in this context, we also observe the accumulation of a type of polysome referred to as a halfmer (Fig. 3c). A halfmer results when a $40 \mathrm{~S}$ complex scans to the start codon of an mRNA that also has an $80 \mathrm{~S}$ ribosome elongating in the open reading frame, and then there is a defect in $60 \mathrm{~S}$ ribosomal subunit joining [38]. Isoamyl alcohol also significantly inhibited translation initiation in wild-type cells (>twofold) $(P<0.05)$. In the evolved strains sSD003 and sSD019 from one evolutionary lineage, translational activity was significantly greater than that of the wild-type strain in the presence of both $n$-hexanol and isoamyl alcohol $(P<0.05)$ (Fig. 3a). For the evolved strains sSD006 and sSD021 from the other lineage, significantly greater translational activity was only observed in $n$-hexanol $(P<0.05)$ (Fig. $3 a)$.

Reconstruction of the $g c d 1-1$ allele from sSD003 and sSD019 in BY4741 (sSD029) relieved the inhibition of translational activity observed with $n$-hexanol and isoamyl alcohol in a control strain (sSD053) carrying $G C D 1^{+}$(Fig. 3b, c) $(P<0.05)$. Similarly, reverseengineering of the sui2-2 allele from sSD021 in BY4741 (sSD056) relieved the inhibition of translation initiation observed in sSD055 carrying SUI2 ${ }^{+}$(Fig. 3b, c) $(P<0.05)$. In contrast to these results, the introduction of the $g c d 7-2$ allele had no effect on the inhibition of translational activity observed with $n$-hexanol and isoamyl alcohol (Fig. 3b). Taken together, these results suggest that the D85E mutation in Gcd1p and the D77Y mutation in Sui2p relieve the inhibition of translation initiation caused by $n$-hexanol and isoamyl alcohol. 

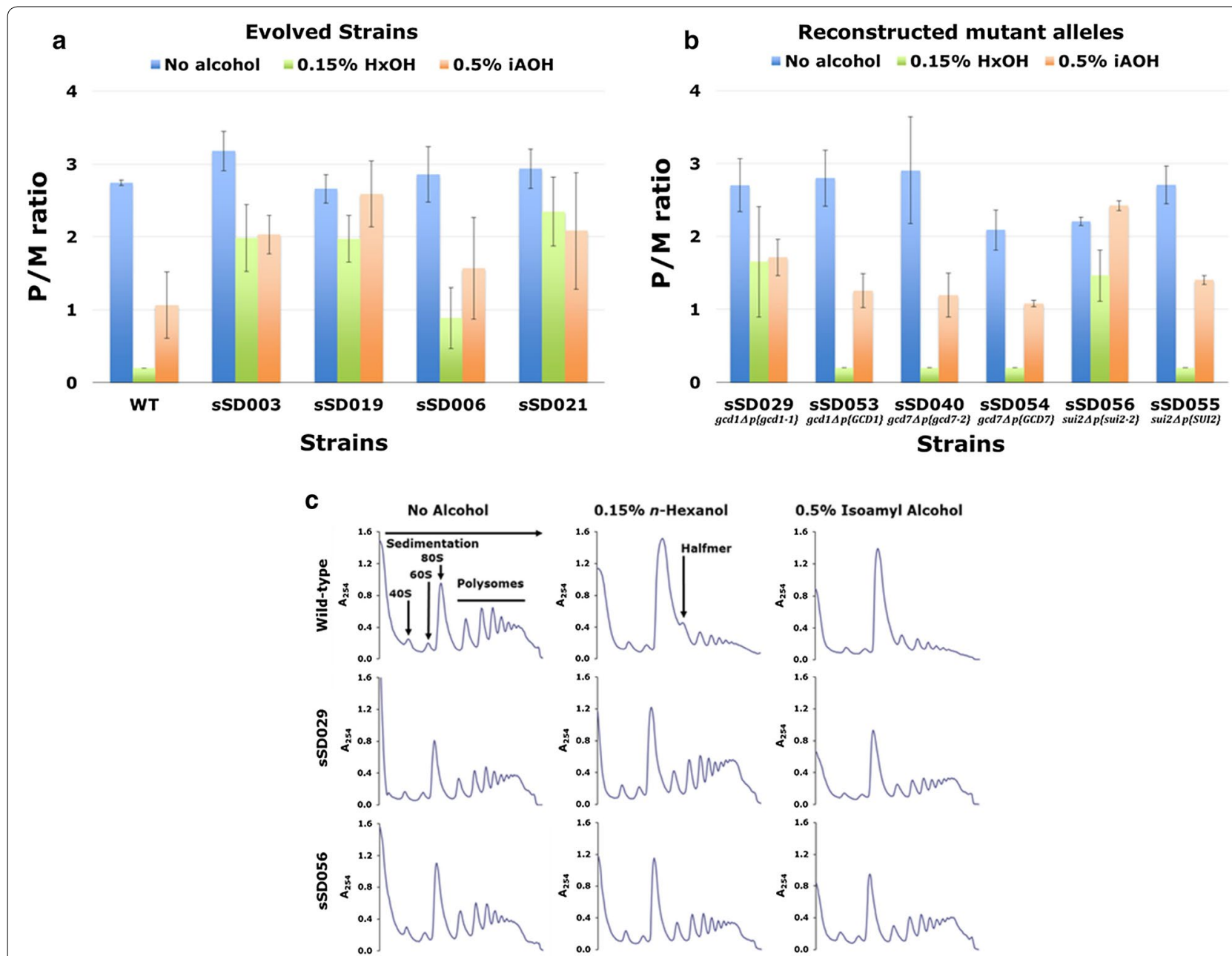

Fig. 3 Mutations in Gcd1p and Sui2p relieve inhibition of translation initiation by $n$-hexanol and isoamyl alcohol. All strains were grown in YPD to an $\mathrm{OD}_{600}$ of $~ 1 \mathrm{AU}$. $n$-Hexanol $(0.15 \%)$ or isoamyl alcohol $(0.5 \%)$ were then added and the cultures incubated for a further 30 min. Polysomes were analyzed as described in "Methods". The P/M ratio was determined for $\mathbf{a}$ WT and evolved strains, and $\mathbf{b}$ strains with the reconstructed mutant alleles (and wild-type genes as controls). Values are mean \pm standard deviation from three independent cultures. c Representative polysome profiles for WT, and the reconstructed gcd1-1 (sSD029) and sui2-2 (sSD056) strains. The 40S (small ribosomal unit), 60S (large ribosomal unit), 80S (monosome), halfmer and polysome peaks are labeled

\section{Amino-acid substitutions of D85 of Gcd1p and D77 of Sui2p modulate $n$-hexanol tolerance}

Importantly, we found that when the mutant alleles gcd1-1 and sui2-2 were individually introduced into cells containing a chromosomal wild-type copy of the respective gene, the $n$-hexanol tolerance phenotype was still observed (Fig. 4). We exploited this fact to investigate the effect of all possible amino-acid substitutions at positions D85 in Gcd1p and D77 Sui2p D77 on $n$-hexanol, $n$-pentanol, and $n$-heptanol tolerance. We reasoned that the evolved strains give us insight into the important residues for tolerance, but all amino acids may not have been sampled during the ALE. All of the remaining 18 naturally occurring amino acids were substituted at the desired site in each protein. The growth of the resulting strains in the presence of $0.13 \%$ $n$-hexanol, $0.5 \% n$-pentanol, and $0.05 \% n$-heptanol is shown in Fig. 5 . The average specific growth rate $\left(\mu_{\text {avg }}\right)$ values for the Gcd1p mutants in $0.13 \% n$-hexanol varied over an $\sim$ fourfold range $\left(0.21-0.05 \mathrm{~h}^{-1}\right)$, both above, and surprisingly, below the value obtained for the wild-type protein $\left(0.11 \mathrm{~h}^{-1}\right)$ (Fig. $\left.5 \mathrm{a}\right)$. A similar pattern was observed in $0.5 \% n$-pentanol $(\sim 3$-fold range of $\mu_{\text {avg }}$ from 0.16 to $0.05 \mathrm{~h}^{-1}$ with a wild-type value of $0.08 \mathrm{~h}^{-1}$ ) (Fig. 5c). The growth rates which were significantly lower than that for the wild-type protein $(P<0.05)$ may suggest that the expression of these Gcd1p mutants was itself toxic to yeast cells. Growth 




Fig. 4 The $n$-hexanol tolerance phenotype conferred by the gcd1-1 and sui2-2 alleles is dominant in BY4741. Strains were grown in $10 \mathrm{ml} \mathrm{SD}$ medium lacking uracil in the presence and absence $0.12 \% n$-hexanol. Values are mean \pm standard deviation from three independent cultures rates for the Gcd1p mutants in $0.05 \% n$-heptanol varied over a $\sim 2$-fold range $\left(0.18-0.08 \mathrm{~h}^{-1}\right)$ with the wild-type protein having the lowest value (Fig. 5e). Interestingly, in all three alcohols, the D85E substitution, which was identified in the evolved strains sSD003 and sSD019, resulted in the highest level of tolerance; no other substitution led to significantly greater alcohol tolerance. The amino-acid substitutions in Gcd1p that confer increased tolerance to alcohols do not share any chemical or structural property that might readily account for why they do so. Substitutions of aliphatic, hydrophobic amino-acids (leucine, isoleucine, and valine) produced mutants that were at the high end of the tolerance spectrum, but so did the introduction of the polar aminoacid lysine, which resulted in a change in the charge at this position.

Tolerance levels for the Sui2p mutants in $0.13 \%$ $n$-hexanol, $0.5 \% n$-pentanol, and $0.05 \% n$-heptanol varied over a 1.6 -fold range $\left(0.21-0.13 \mathrm{~h}^{-1}\right), 1.8$-fold range $\left(0.14-0.08 \mathrm{~h}^{-1}\right)$, and twofold range $\left(0.16-0.08 \mathrm{~h}^{-1}\right)$, respectively, and in each case, the wild-type protein had the lowest, or second lowest tolerance (Fig. 5b, d, f). Once again, in all three alcohols, the D77Y substitution, which was identified in the evolved strain SSD021, resulted in the highest level of tolerance with no other substitution giving rise to significantly greater alcohol tolerance. As for the Gcd1p mutants, no single aminoacid property readily accounts for the improved alcohol tolerance observed with the Sui2p mutants. In fact, nearly all substitutions at D77 in Sui2p resulted in significantly improved alcohol tolerance.

\section{Discussion}

A significant challenge in the development of cost-competitive production processes for advanced higher alcohol biofuels in S. cerevisiae is that these compounds are often highly toxic to yeast cells. This problem provided the motivation for the current work in which we sought to identify genetic targets that can be exploited in tolerance engineering and strain development for biofuel production. S. cerevisiae strains adapted for growth in $n$-hexanol concentrations as high as $0.2 \%$ were isolated through ALE. The evolved strains showed increased tolerance to $n$-hexanol which was most pronounced in the concentration range $0.15-0.2 \%$ (Fig. $1 \mathrm{~b}$ ) where the parental strain was unable to grow. Although difficult to evaluate the significance of these tolerance increases from the point of view of the contribution they might make to $n$-hexanol production, a recent study of $n$-butanol production in S. cerevisiae demonstrated that a strain that was more resistant to the toxic effects of butanol produced significantly more of it [6]. Future studies will have to establish whether the increased tolerance of yeast cells to $n$-hexanol, and other medium-chain alcohols, afforded by these mutations leads to increased titers of target bioalcohols in production strains.

Remarkably, in both of the two independent evolutionary lineages of $n$-hexanol-tolerant S. cerevisiae established in the current study, mutations in translation initiation proteins were identified. One mechanism for the toxicity of the fusel alcohols ( $n$-butanol, isobutanol, 2-methylbutan-2-ol, and isoamyl alcohol) in S. cerevisiae involves inhibition of translation initiation due to perturbation in eIF2B activity that lead to reduced levels of the eIF2-GTP Met-tRNA ${ }^{i}$ ternary complex $[14,15]$. Moreover, fusel alcohol-dependent translational inhibition is modulated by mutations in all five eIF2B subunits (subunits of the catalytic subcomplex: $\gamma(\operatorname{Gcd} 1 \mathrm{p})[14,15]$ and $\varepsilon$ (Gcd6p) [15, 39]; subunits of the regulatory subcomplex; $\alpha$ (Gcn3p) [15], $\beta$ (Gcd7p) [39], and $\delta(G c d 2 p)$ [14]). In one of our lineages, a D85E mutation in Gcd1p occurred during the first 30-day round of adaptive evolution. Reverse-engineering of this mutation demonstrated that it conferred $n$-hexanol tolerance to BY4741 (Fig. 2a, b). Polysome profiling revealed that $n$-hexanol does indeed inhibit translation initiation as evidenced by a dramatic decrease in the $\mathrm{P} / \mathrm{M}$ ratio (as compared to a no alcohol control) and the appearance of halfmers (Fig. 3a, c), and that reconstruction of the D85E Gcd1p mutation in BY4741 significantly attenuated inhibition of translational activity (Fig. 3b, c). These results suggest that $n$-hexanol likely shares the same eIF2B-mediated mechanism of translational inhibition as the fusel alcohols. Remarkably, examination of all possible amino-acid substitutions at position D85 in Gcd1p revealed that the 


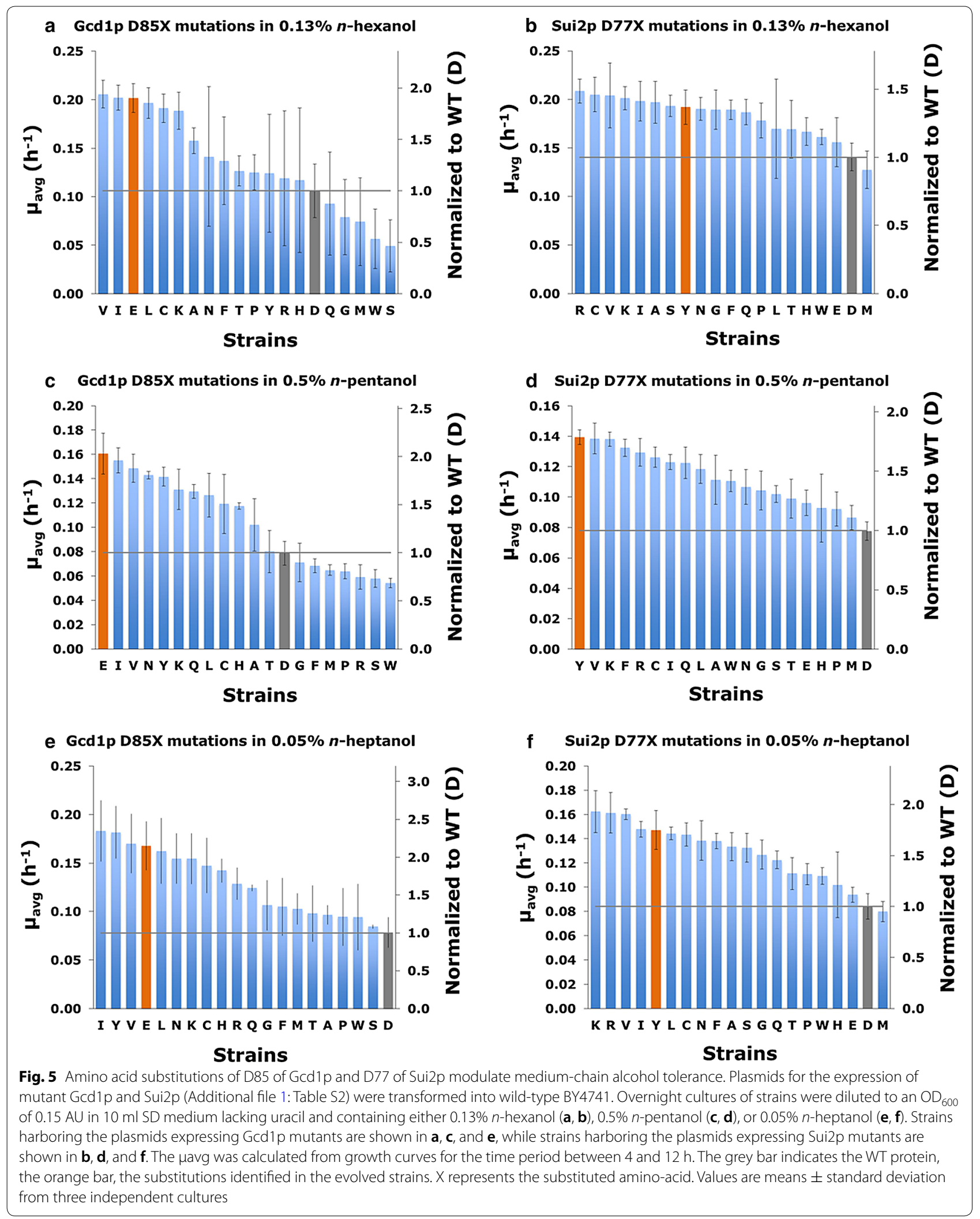


evolved mutation D85E resulted in the highest possible level of tolerance to medium-chain alcohols (Fig. 5a, c, e). In contrast to the D85E eIF2By mutation identified here, other mutations in this subunit $(g c d 1-\mathrm{P} 180 \mathrm{~S}, \mathrm{gcd} 1$ C483W, and $g c d 1-101$ ) were found to increase sensitivity to alcohol [14].

In our second evolutionary lineage, mutations occurred in two other translation initiation proteins, and the more significant of the two, a D77Y mutation in Sui2p, the $\alpha$ subunit of the translation initiation factor eIF2 (eIF2 $\alpha$ ), occurred during the second 30-day round of ALE. Reverse-engineering of this mutation demonstrated that it too conferred $n$-hexanol tolerance to BY4741 (Fig. 2e, f) by relieving inhibition of translation initiation (Fig. 3b, c). And as was found for our eIF2By mutation, site-saturation mutagenesis experiments showed that the evolved D77Y mutation in Sui2p resulted in the highest possible level of tolerance to medium-chain alcohols (Fig. 5b, d, f), highlighting the effectiveness of ALE for tolerance engineering. To our knowledge, this is the first report of a mutation in eIF2 that modulates alcohol-dependent translational inhibition, and the first to suggest eIF $2 \alpha$ as a genetic target for tolerance engineering to mediumchain alcohols in S. cerevisiae.

The remaining translation initiation protein mutation in our second lineage was R56C in eIF2B $\beta$, which occurred in the first 30-day round of ALE. Although the evolved strain sSD006, which contains this mutation as the sole mutation detected, exhibited improved tolerance to $0.15 \% n$-hexanol (Fig. 1a) [and 0.05\% 3-octanol (Table 2)] and had significantly higher translational activity in the presence of $n$-hexanol than the WT strain (Fig. 3a), we were unable to show that the mutation, when reconstructed in BY4741, actually conferred $n$-hexanol tolerance (Fig. 2c, d) or affected $n$-hexanoldependent inhibition of translation (Fig. 3b). This may have been due to the fact, noted earlier, that full complementation of BY4741 $g c d 7 \Delta$ by plasmid-borne $g c d 7-2$ was most likely not achieved. If too little of the mutant Gcd7p was expressed in the reconstructed strain, this may itself have led to lower eIF2B activity. Two mutations in eIF2B $\beta, g c d 7-201$ and $g c d 7-\mathrm{V} 341 \mathrm{D}$, make growth more sensitive to $n$-butanol [39], supporting the notion that the eIF2B $\beta^{\mathrm{R} 56 \mathrm{C}}$ mutant identified here may play a role in $n$-hexanol tolerance.

The evolved strains reported in this paper (particularly, sSD003, sSD019, and sSD021) showed improved tolerance to a wide range of alcohols (Table 2), suggesting that the mutations in the translation initiation proteins identified here (particularly D85E in Gcd1p and D77Y in Sui2p) may play a role in alleviating the impact of these alcohols on the translational apparatus, and may be useful in work directed at producing these alcohols in S. cerevisiae. An interesting observation in these data was that the strain sSD003 containing the D85E mutation in Gcd1p and the strain sSD021 with the D77Y mutation in Sui2p behaved differently with respect to growth in $n$-propanol and butanol (Table 2). sSD003 showed improved tolerance to these alcohols whereas sSD021 was more sensitive to them. This result suggests that these mutations may prove useful in probing the mechanism by which these alcohols interact with eIF2/eIF2B to alter their activity.

In the absence of any information on where fusel alcohols and other medium-chain alcohols bind to eIF2B, and perhaps also to eIF2, the specific roles of the translation initiation protein mutations identified here in alleviating alcohol-dependent translational inhibition remain unresolved and will require further work. Nevertheless, the observation that a mutation in eIF $2 \alpha$, in addition to those in eIF2B, can rescue alcohol-dependent translational inhibition, suggests that alcohols may perturb eIF2/eIF2B activity by binding at the interface of the proteins, and in so doing, alter their interaction. Recently, the crystal structure of the Schizosaccharomyces pombe eIF2B was determined [40], and studies aimed at experimentally mapping the interaction surfaces between eIF2 and eIF2B were performed $[40,41]$. The locations of the homologous residues in the S. pombe eIF2By (E81, P156, and C411) corresponding to those found to modulate alcohol sensitivity in the S. cerevisiae eIF2By (D85, P180, C483, respectively) are shown in the $S$. pombe eIF2B structure in Additional file 1: Figure S5. Also shown in the red hatched box are the location of surfaces of eIF2B $\gamma$ and eIF2B $\varepsilon$ found to make contacts with eIF2 $\gamma$, the eIF2 subunit to which GTP is bound [40, 41]. E81 and P156 are located in the vicinity of this interaction surface, whereas C411 is further away. The homologous residue corresponding to R56 in the $S$. pombe eIF2B $\beta$ is R47 which is located on the surface of the subunit, but outside of the areas shown to be involved in interactions with eIF2 $\gamma$ (red hatched box) and eIF $2 \alpha$ (orange hatched box). The residue corresponding to V341 in eIF2B $\beta$, I358, is not surface located.

The structure of the N-terminal region of the $S$. cerevisiae eIF2 $\alpha$ has also been determined by X-ray crystallography [42]. Mutational analysis of eIF2 $\alpha$ has identified two non-contiguous segments comprising residues E49 and R88 (that form a salt-bridge), in close proximity to the phosphorylation site, S51, and residues K79 and G80, located 30 or more residues away from S51, that may be involved in binding to eIF2B [42, 43] (Additional file 1: Figure S6). Phosphorylation of S51 of eIF2 $\alpha$ enhances its interaction with eIF2B. The alcohol-sensitive D77 residue (D76 in the structure that lacks M1) is well placed to influence eIF $2 \alpha$ binding to eIF2B. 


\section{Conclusions}

Analysis of $n$-hexanol-tolerant $S$. cerevisiae strains isolated by ALE revealed that a mechanism of $n$-hexanol toxicity in yeast is inhibition of translation initiation. Mutations in three translation initiation protein genes, GCD1, GCD7, and SUI2, significantly increased tolerance to $n$-hexanol (and a range of other medium-chain alcohols), and can be exploited in future metabolic engineering efforts for the production of $n$-hexanol and other medium-chain alcohols.

\section{Additional file}

Additional file 1: Figure S1. Representative growth curves for evolved strains in $0.15 \% n$-hexanol. Strains were grown in $10 \mathrm{ml} \mathrm{YPD}+0.15 \%$ $n$-hexanol in capped $50 \mathrm{ml}$ culture tubes. Figure S2. Growth curves for evolved strains in $0.5 \%$ n-pentanol. Figure S3. Growth curves for evolved strains in $0.05 \% n$-heptanol. Figure $\mathbf{S 4}$. Growth curves to examine the impact of Pdr5 in $0.15 \%$ n-hexanol. Figure S5. Structure of S. pombe elF2B with the positions of alcohol-sensitive S. cerevisiae elF2B $\gamma$ and elF2B $\beta$ mutations mapped. The subunit arrangement depicted is of the $a_{2} \beta_{2} \delta_{2}$ hexameric regulatory subcomplex bound to two $\gamma \varepsilon$ dimeric catalytic subcomplexes on its opposite sides [40]. The $a_{-}^{-}, \beta-, \gamma^{-}, \delta$ - and $\varepsilon$-subunits are colored blue, cyan, orange, green and pink, respectively. The positions of mapped alcohol-sensitive S. cerevisiae eIF2By and elF2B 3 mutations are indicated on the elF2Bye subcomplex on the left only. The location of the interfaces for elF2 $\gamma$ and elF2 $a$ binding to elF2B are indicated by the red and orange hatched boxes, respectively $[40,41]$. Figure S6. Structure of S. cerevisiae elF2a (residues 1-175) [42] indicating the phosphorylation site, S51, the alcohol-sensitive D77 (D76 in structure that lacks M1), and residues that have been shown to be important for interaction with elF2B (E49, K79, G80, and R88) $[42,43]$. Table S1. List of primers used in this study. Table S2. List of plasmids for expression of Gcd1p and Sui2p mutants.

\section{Authors' contributions}

SADL designed and carried out adaptive evolution, growth, and polysome experiments, analyzed data, and wrote and edited the manuscript. DAG performed additional growth experiments, analyzed data, and wrote and edited the manuscript. BC performed additional growth experiments and analyzed data. JC and DTE conceived experiments, assisted in experimental design, data analysis and interpretation, and wrote and edited the manuscript. All authors read and approved the final manuscript.

\section{Author details}

${ }^{1}$ Next Interactions, Richmond, CA 94806, USA. ${ }^{2}$ Department of Chemical and Biological Engineering, Northwestern University, 2145 Sheridan Road, Tech E-136, Evanston, IL 60208-3109, USA. ${ }^{3}$ Department of Chemical and Biomolecular Engineering, University of California, Berkeley, CA 94720, USA.

${ }^{4}$ Department of Chemistry, University of California, Berkeley, CA 94720, USA.

\section{Acknowledgements}

The authors would like to thank Han Teng Wong and Jeff Skerker for their help in analyzing sequencing data, Han Teng Wong and Charlotte Abrahamson for their help in designing the figures, and the Cate, Arkin, and Tullman-Ercek labs for valuable discussions of this manuscript.

\section{Competing interests}

The authors declare that they have no competing interests.

\section{Availability of data and materials}

All data generated or analyzed during this study are included in this published article and its additional information files.
Consent for publication

Not applicable.

Ethics approval and consent to participate

Not applicable.

Funding

This work was funded by the Energy Biosciences Institute.

\section{Publisher's Note}

Springer Nature remains neutral with regard to jurisdictional claims in published maps and institutional affiliations.

Received: 29 November 2017 Accepted: 21 March 2018

Published online: 02 April 2018

\section{References}

1. Steen EJ, Chan R, Prasad N, Myers S, Petzold CJ, Redding S, et al. Metabolic engineering of Saccharomyces cerevisiae for the production of $n$-butanol. Microb Cell Fact. 2008;7:36.

2. Sakuragi H, Morisaka H, Kuroda K, Ueda M. Enhanced butanol production by eukaryotic Saccharomyces cerevisiae engineered to contain an improved pathway. Biosci Biotechnol Biochem. 2015;79:314-20.

3. Krivoruchko A, Serrano-Amatriain C, Chen Y, Siewers V, Nielsen J. Improving biobutanol production in engineered Saccharomyces cerevisiae by manipulation of acetyl-CoA metabolism. J Ind Microbiol Biotechnol. 2013:40:1051-6.

4. Si T, Luo Y, Xiao H, Zhao H. Utilizing an endogenous pathway for 1-butanol production in Saccharomyces cerevisiae. Metab Eng. 2014;22:60-8

5. Branduardi P, Longo V, Berterame NM, Rossi G, Porro D. A novel pathway to produce butanol and isobutanol in Saccharomyces cerevisiae. Biotechnol Biofuels. 2013;6:68.

6. Swidah R, Wang H, Ahmed HZ, Pisanelli AM, Persaud KC, Grant CM, et al. Butanol production in S. cerevisiae via a synthetic ABE pathway is enhanced by specific metabolic engineering and butanol resistance. Biotechnol Biofuels. 2015;8:97.

7. Chen X, Nielsen KF, Borodina I, Kielland-Brandt MC, Karhumaa K. Increased isobutanol production in Saccharomyces cerevisiae by overexpression of genes in valine metabolism. Biotechnol Biofuels. 2011:4:21.

8. Avalos JL, Fink GR, Stephanopoulos G. Compartmentalization of metabolic pathways in yeast mitochondria improves the production of branched-chain alcohols. Nat Biotechnol. 2013;31(4):335-41.

9. Ohto C, Muramatsu M, Obata S, Sakuradani E, Shimizu S. Overexpression of the gene encoding HMG-CoA reductase in Saccharomyces cerevisiae for production of prenyl alcohols. Appl Microbiol Biotechnol. 2009;82:837-45.

10. Madeira A, Leitão L, Soveral G, Dias P, Prista C, Moura T, et al. Effect of ethanol on fluxes of water and protons across the plasma membrane of Saccharomyces cerevisiae. FEMS Yeast Res. 2010;10(3):252-8.

11. González-Ramos D, van den Broek M, van Maris AJA, Pronk JT, Daran J-MG. Genome-scale analysis of butanol tolerance in Saccharomyces cerevisiae reveal an essential role of protein degradation. Biotechnol Biofuels. 2013;6:48.

12. Dunlop MJ. Engineering microbes for tolerance to next-generation biofuels. Biotechnol Biofuels. 2011;21(4):32.

13. Gaskova D, Plasek J, Zahumensky J, Benesova I, Buriankova L, Sigler K. Alcohols are inhibitors of Saccharomyces cerevisiae multidrug-resistance pumps Pdr5p and Snq2p. FEMS Yeast Res. 2013;13(8):782-95.

14. Ashe MP, Slaven JW, DeLong SK, Ibrahimo S, Sachs AB. A novel elF2Bdependent mechanism of translational control in yeast as a response to fusel alcohols. EMBO J. 2001;20(22):6464-74.

15. Taylor EJ, Campbell SG, Griffiths CD, Reid PJ, Slaven JW, Harrison RJ, et al. Fusel alcohols regulate translation initiation by inhibiting elF2B to reduce ternary complex in a mechanism that my involve altering the integrity and dynamics of the elF2B body. Mol Biol Cell. 2010;21:2202-16. 
16. Merrick WC. Eukaryotic protein synthesis: still a mystery. J Biol Chem. 2010;285(28):21197-201.

17. Hinnebusch AG. Translational regulation of GCN4 and the general amino acid control of yeast. Annu Rev Microbiol. 2005;59:407-50.

18. Li X, Pei G, Liu L, Chen L, Zhang W. Metabolomic analysis and lipid accumulation in a glucose tolerant Crypthecodinium cohnii strain obtained by adaptive laboratory evolution. Bioresour Technol. 2017;235:87-95.

19. Caspeta L, Chen Y, Ghiaci P, Feizi A, Buskov S, Hallström BM, et al. Altered sterol composition renders yeast thermotolerant. Science. 2014;346(6205):75-8.

20. Ghiaci P, Norbeck J, Larsson C. Physiological adaptations of Saccharomyces cerevisiae evolved for improved butanol tolerance. Biotechnol Biofuels. 2013;6:101.

21. Brennan TCR, Williams TC, Schulz BL, Palfreyman RW, Krömer JO, Nielsen LK. Evolutionary engineering improves tolerance for replacement jet fuels in Saccharomyces cerevisiae. Appl Environ Microbiol. 2015;81(10):3316-24.

22. Turanlı-Yıldız B, Benbadis L, Alkım C, Sezgin T, Akşit A, Gökçe A, et al. In vivo evolutionary engineering for ethanol-tolerance of Saccharomyces cerevisiae haploid cells triggers diploidization. J Biosci Bioeng. 2017;124(3):309-18.

23. Sambrook J, Fritsch EF, Maniatis T. Molecular cloning: a laboratory manual. 2nd ed. Cold Spring Harbor: Cold Spring Harbor Laboratory Press; 1989.

24. Oldenburg KR, Vo KT, Michaelis S, Paddon C. Recombination-mediated PCR-directed plasmid construction in vivo in yeast. Nucleic Acids Res. 1997;25:451-2.

25. Schiestle RH, Gietz RD. High efficiency transformation of intact cells using single stranded nucleic acids as a carrier. Curr Genet. 1989;16:339-46.

26. Lee ME, Aswani A, Han AS, Tomlin CJ, Dueber JE. Expression-level optimization of a multi-enzyme pathway in the absence of a high-throughput assay. Nucleic Acids Res. 2013;41 (22):10668-78.

27. Jones M. Isolation of plasmid DNA from yeast using the QIAprep ${ }^{\circledR}$ Spin Miniprep Kit. 2001. https://www.qiagen.com/us/resources/resourcede tail?id=5b59b6b3-f11d-4215-b3f7-995a95875fc0\&lang=en. Accessed 15 Jan 2014.

28. Erdeniz N, Mortensen UH, Rothstein R. Cloning-free PCR-based allele replacement methods. Genome Res. 1997;7:1174-83.

29. Bolger AM, Lohse M, Usadel B. Trimmomatic: a flexible trimmer for illumina sequence data. Bioinformatics. 2014;30:2114-20.

30. Li H. Aligning sequence reads, clone sequences and assembly contigs with BWA-MEM. 2013. arXiv: 1303.3997v1 [q-bio.GN].
31. Garrison E, Marth G. Haplotype-based variant detection from short-read sequencing. arXiv preprint arXiv. 2012; 1207.3907 [q-bio.GN].

32. Quinlan AR. BEDTools: the Swiss-army tool for genome feature analysis. Curr Protoc Bioinform. 2014;47:11-2.

33. Cingolani P, Platts A, le Wang L, Coon M, Nguyen T, Wang L, et al. A program for annotating and predicting the effects of single nucleotide polymorphisms, SnpEff: SNPs in the genome of Drosophila melanogaster strain w1118; iso-2; iso-3. Fly. 2012;6(2):80-92.

34. Pospísek M, Valásek L. Polysome profile analysis—yeast. Methods Enzymol. 2013;530:173-81.

35. Van Dyk TK. Yeast with increased butanol tolerance involving a multidrug efflux pump gene. US Patent 8614085 B2, December 24, 2013.

36. Winzeler EA, Shoemaker DD, Astromoff A, Liang H, Anderson K, Andre B, et al. Functional characterization of the Saccharomyces cerevisiae genome by gene deletion and parallel analysis. Science. 1999;285:901-6.

37. Wagner M, Doehl K, Schmitt L. Transmitting the energy: interdomain cross-talk in Pdr5. Biol Chem. 2017;398(2):145-54.

38. Lee JH, Pestova TV, Shin BS, Cao C, Choi SK, Dever TE. Initiation factor elF5B catalyzes second GTP-dependent step in eukaryotic translation initiation. PNAS. 2002;99(26):16689-94.

39. Richardson JP, Mohammad SS, Pavitt GD. Mutations causing childhood ataxia with central nervous system hypomyelination reduce eukaryotic initiation factor 2B complex formation and activity. Mol Cell Biol. 2004:24:2352-63.

40. Kashiwagi K, Takahashi M, Nishimoto M, Hiyama TB, Higo T, Umehara T, et al. Crystal structure of eukaryotic translation initiation factor 2B. Nature. 2016:531:122-5

41. Kashiwagi $\mathrm{K}$, Ito T, Yokoyama S. Crystal structure of elF2B and insights into elF2-elF2B interactions. FEBS J. 2017:284:868-74.

42. Dhaliwal S, Hoffman DW. The crystal structure of the N-terminal region of the alpha subunit of translation initiation factor 2 (elF2a) from Saccharomyces cerevisiae provides a view of the loop containing serine 51 , the target of the elF2alpha-specific kinases. J Mol Biol. 2003;334:187-95.

43. Vazquez de Aldana CR, Dever TE, Hinnebusch AG. Mutations in the a; subunit of eukaryotic translation initiation factor 2 that overcome the inhibitory effects of elF-2a phosphorylation on translation initiation. Proc Natl Acad Sci. 1993;90:7215-9.

\section{Submit your next manuscript to BioMed Central and we will help you at every step:}

- We accept pre-submission inquiries

- Our selector tool helps you to find the most relevant journal

- We provide round the clock customer support

- Convenient online submission

- Thorough peer review

- Inclusion in PubMed and all major indexing services

- Maximum visibility for your research

Submit your manuscript at www.biomedcentral.com/submit
BioMed Central 